\title{
Evaluation of the prostatic artery origin using computed tomography angiography
}

\author{
Emre Can Çelebioğlu ${ }^{1}$, Sinem Akkaşoğlu², Selma Çalışkan², Ceren Günenç Beşer ${ }^{3}$, Tanzer Sancak ${ }^{1}$ \\ ${ }^{1}$ Department of Radiology, School of Medicine, TOBВ University, Ankara, Turkey \\ ${ }^{2}$ Department of Anatomy, School of Medicine, Ankara Ylldirm Beyazut University, Ankara, Turkey \\ ${ }^{3}$ Department of Anatomy, School of Medicine, Hacettepe University, Ankara, Turkey
}

\begin{abstract}
Objectives: Radiological anatomy of the prostatic artery is important for any kind of surgical or interventional procedure related to the prostatic region. The aim of this study was to define prostatic arterial anatomy that is critical for both urologists dealing with prostatic interventions and interventional radiologists dealing with prostatic arterial embolization.

Methods: Computed tomography angiography (CTA) is the gold standard for visualizing pelvic arterial anatomy. In this study, morphometric analyses were performed with CTA in 121 patients (41-89 years) retrospectively. The diameters and origins of 242 prostatic arteries were evaluated.

Results: Average diameter of the right prostatic artery was $0.9-2.4 \mathrm{~mm}$; in 39\% of the patients the artery originated from the inferior vesical artery. The other origin patterns on the right side were the internal pudendal artery (36\%), gluteopudendal trunk (7.4\%), obturator (5,8\%), inferior gluteal (3.3\%), middle rectal $(0.8 \%)$, umbilical $(0.8 \%)$ and inferior rectal $(0.8 \%)$ arteries, and the vesical trunk $(0.8 \%)$. Average diameter of the left prostatic artery was $0.9-2.7 \mathrm{~mm}$; in $36 \%$ of the patients the artery originated from the inferior vesical artery. Other origin patterns revealed for the left side were the internal pudendal artery (31\%), obturator artery (13\%), gluteopudendal trunk (9.1\%), vesical trunk $(2.5 \%)$ and inferior gluteal artery $(2.5 \%)$.

Conclusion: CTA is crucial for better understanding of the prostatic arterial anatomy and preventing the complications in surgical or interventional procedures such as prostatic arterial embolization, especially for atherosclerotic patients. The data obtained in this study is significant for determination of pelvic vascular disorders.
\end{abstract}

Keywords: anatomy; computed tomography angiography; embolization; prostatic artery; variation

Anatomy 2018;12(3):140-144 @2018 Turkish Society of Anatomy and Clinical Anatomy (TSACA)

\section{Introduction}

Benign prostatic hyperplasia (BPH) is one of the most frequent health problems in males that impairs the quality of life in sixth and seventh decades. ${ }^{[1,2]}$ Medications such as 5$\alpha$-reductase inhibitors and selective $\alpha$ blockers may help decreasing the voiding symptoms in patients with $\mathrm{BPH}$, but the curative treatment option is surgery. Because of the comorbidities in the elderly, open surgical prostatectomy is considered high risk. Alternative treatments are the minimally invasive transurethral therapies and the prostatic artery embolization (PAE). ${ }^{[1,3]}$
In previous times, PAE was used to stop the bleeding after radical prostatectomy or prostate biopsy. Reduced prostate volume after PAE was reported in experimental animal studies and case series in the literature. ${ }^{[1]} \mathrm{PAE}$ has been shown as a minimal invasive technique that can serve as a strong alternative for surgery at $\mathrm{BPH}$ with low morbidity rates. ${ }^{[1]}$ Maintenance of sexual functions and eliminations of daily $\mathrm{BPH}$ medications are additional benefits of this procedure. $^{[4]}$

Knowledge of the radiological anatomy of the prostatic arteries (PAs) is very important before any kind of sur-

This study was presented as an oral presentation at the 19th National Anatomy and 1st International Mediterranean Anatomy Congress, September 6-9, 2018, Konya, Turkey, and this presentation was elected as the best oral presentation in the congress. 
gical or interventional procedure. Computed tomography angiography (CTA) is the gold standard for showing pelvic arterial anatomy. ${ }^{[5]}$ Prostate surgery may also cause erectile dysfunction due to the condition of the cavernous nerve and vascular variations such as accessory pudendal artery. ${ }^{[6]}$ CTA presents a detailed anatomical knowledge of vascular variations, helps to prevent complications and ensures optimal postoperative sexual function. ${ }^{[6]}$ Variations of the PA have critical importance and should be well known by the interventional radiologists who are dealing with PAE to prevent ischemic damage of bladder, penis or rectum after embolization. ${ }^{[5,7]}$ Preprocedural CTA could be a good option to demonstrate pelvic arterial anatomy before PAE. ${ }^{[7]}$

Pelvic arterial anatomy and most common variations in males were previously studied. There are cadaveric and radiological studies in the literature enlightening the vascular supply of pelvic organs. ${ }^{[7-9]}$

In this study, we aimed to define and classify the PA variations in Turkey by CTA and compare the differences of PA anatomy with earlier studies performed in other populations.

\section{Materials and Methods}

Ethics committee approval was received by TOBB ETU Faculty of Medicine Clinical Research Ethics Committee (number: KAEK 118/031). A total of 121 patients were included in the study and were evaluated retrospectively by CTA. Each pelvic side was considered seperately, and the diameters and origins of 242 PAs were reported. The ages of the patients were between 41-89 (mean: $65.29 \pm 10.66)$ years. The diameters of the right and left PAs were measured and the mean diameter for each side was determined.

The CTA images of the cases were obtained from the archive system of TOBB ETU University. CTA examinations were obtained with Philips Ingenuity 128 slice computerized tomography device (Philips Medical Systems, Cleveland, OH, USA). Patient dose parameters were adjusted automatically by the device. $0.8 \mathrm{~mm}$ slice thickness and a pitch value of 1 were used with a 300/100 $\mathrm{ml}$ or 350/100 $\mathrm{ml}$ non-ionic iodinated contrast material. We did not administer any IV or sublingual vasodilators before the angiography procedure. Patients in this study population did not use any kind of medications for prostate disease symptoms. CT scans were made at an area between abdominal aorta to common femoral or superficial femoral arteries near femoral heads. Pelvic arterial anatomy was evaluated with the device software (Philips Intellispace Portal 9.0; Philips Medical Systems, Cleveland, OH, USA) and post-process procedures like slice thickness arrangement, maximum intensity projection (MIP), multiplanar reconstructions (MPR) and 3D reconstructions were made by the radiologist with the software, to reach at the utmost level for evaluating the pelvic anatomy of each patient.

Descriptive statistics were used to get the frequency of each vessel category. Statistical Package for Social Sciences (SPSS for Windows, version 21.0; SPSS Inc., Armonk, NY, USA) was used for analyses. Differences between two means were tested by using t-test and $\mathrm{p}<0.05$ was considered as statistical significant.

\section{Results}

121 patients who underwent CTA imaging for various indications were included in this study. Each pelvic side was considered seperately, and the diameters and origins of 242 PAs were reported.

The mean diameter of the right and left PAs were measured as $1.56 \pm 0.35(0.9-2.4) \mathrm{mm}$ and $1.54 \pm 0.3$ (0.9-2.7) mm respectively. The minimum, maximum and mean values of the diameters of the right and left PAs are shown in Figure 1.

In this study, a total of 242 PAs originating from nine different sources were reported. Of the 121 right PAs, 47 (39\%) were found to originate from the inferior vesical artery. Forty-three of the cases (36\%) originated from the internal pudendal artery, $9(7.4 \%)$ from the gluteo-pudendal trunk, 7 (5.8\%) of from the obturator artery, 4 (3.3\%) from the inferior gluteal artery, $1(0.8 \%)$ from the middle

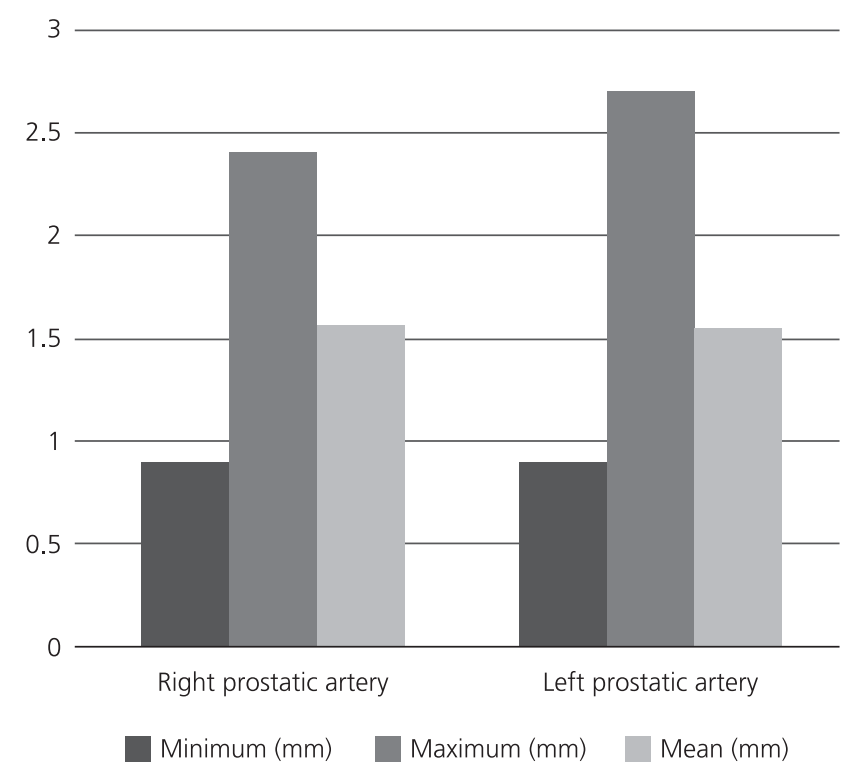

Figure 1. Diameter of the right and left prostatic artery. 
rectal artery, $1(0.8 \%)$ from the umbilical artery, $1(0.8 \%)$ from the vesical trunk and $1(0.8 \%)$ from the inferior rectal artery (Figures $\mathbf{2}$ and $\mathbf{3}$; Table $\mathbf{1}$ ).

Of the 121 left PAs, 43 (36\%) originated from the inferior vesical artery. Thirty-eight $(31 \%)$ cases originated from the internal pudendal artery, $16(13 \%)$ cases from the obturator artery, $11(9.1 \%)$ from the gluteo-pudendal trunk, 3 (2.5\%) from the vesical trunk and $3(\% 2.5)$ from the inferior gluteal artery (Figures 2 and 3; Table 2).

The origins of right and left PAs are summarized in Tables 1 and 2.

In this study, accessory prostatic arteries were also evaluated. Accessory prostatic arteries were found in 10 cases; 8 of them were on the left and 2 of them were on right side.

\section{Discussion}

Because of the indistinguishable fat and small nerves and vessels, it is difficult to evaluate the pelvic vascular anatomy in cadavers. ${ }^{[10-12]}$ Besides, cadaveric dissection procedure is likely to damage the relevant structures. ${ }^{[10]}$ Radiologic images present us detailed information about anastomoses, variations and dominance of arteries supplying the prostate. These are important parameters when planning PAE to prevent penis, rectum and bladder from ischemia related to non-target embolization. ${ }^{[10]}$ CTA images are used in this study because of the ability to receive more detailed information about branching patterns of vessels in large patient series in a short time period.

PAs may be depicted by cadaver dissection and imaging modalities such as CTA, digital subtraction angiography (DSA), MR angiography (MRA) and cone beam CT. ${ }^{[5,7-12]}$ Because CTA and MRA are non-invasive procedures, they allow better planning of PAE than digital angiography ${ }^{[5]}$ Besides, Bilhim et al ${ }^{[5]}$ claimed that CTA provides more detailed anatomy than MRA. DSA is sometimes incapable of identifying the PA origin because of superimpositions. ${ }^{[10]}$ Cone beam CT has been considered as a good option for better understanding of the pelvic arterial anatomy, with less contrast media and less X-ray doses with similar results, in contrast to CTA. However, it is still an invasive technique that should be done with an arterial puncture intra-procedurally at an angiography suite. It has recently been shown that there are no subjective advantages of cone beam CT over CTA. Besides having a CTA before PA, embolization may affect the puncture site, catheter selection or even abortion of the embolization procedure due to the arterial anatomy or atherosclerotic disease ${ }^{[10,13]}$ CTA is still
Table 1

Origin of the right prostatic artery.

\begin{tabular}{lcc}
\hline Origin & $\mathbf{n}$ & Ratio (\%) \\
\hline Internal pudendal artery & 43 & 36 \\
Inferior vesical artery & 47 & 39 \\
Middle rectal artery & 1 & 0.8 \\
Obturator artery & 7 & 5.8 \\
Gluteo-pudendal artery & 9 & 7.4 \\
Inferior gluteal artery & 4 & 3.3 \\
Umblical artery & 1 & 0.8 \\
Vesical trunk & 1 & 0.8 \\
Inferior rectal artery & 1 & 0.8 \\
\hline
\end{tabular}

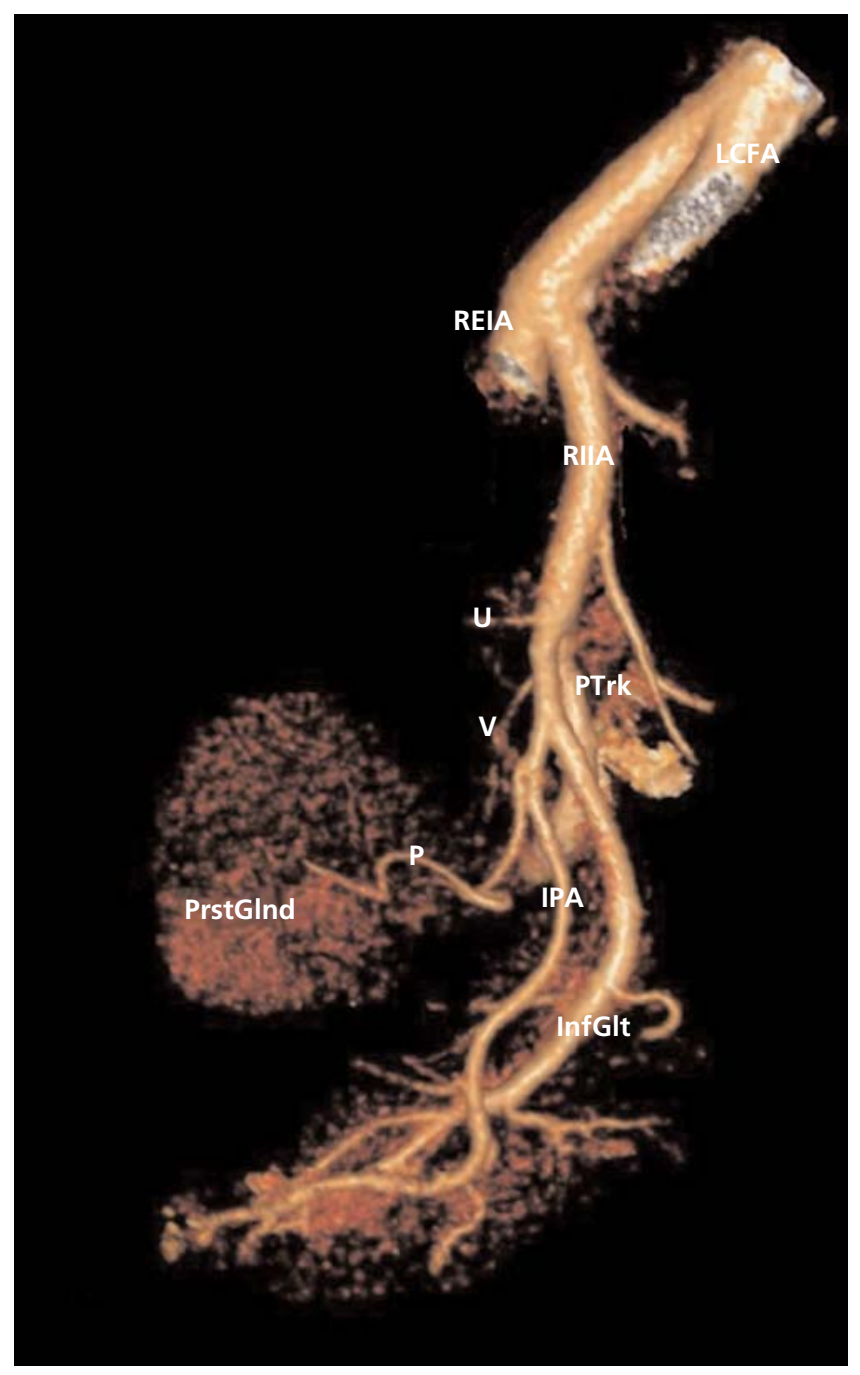

Figure 2. 3D rendered images of right internal iliac artery anterior division. InfGlt: inferior gluteal artery; IPA: internal pudendal artery; LCIA: left common iliac artery; P: prostatic artery; REIA: right external iliac artery; RIIA: right internal iliac artery; U: umblical artery; PrstGInd: prostatic gland; PTrk: posterior trunk; V: vesical artery. [Color figure can be viewed in the online issue, which is available at www.anatomy.org.tr] 

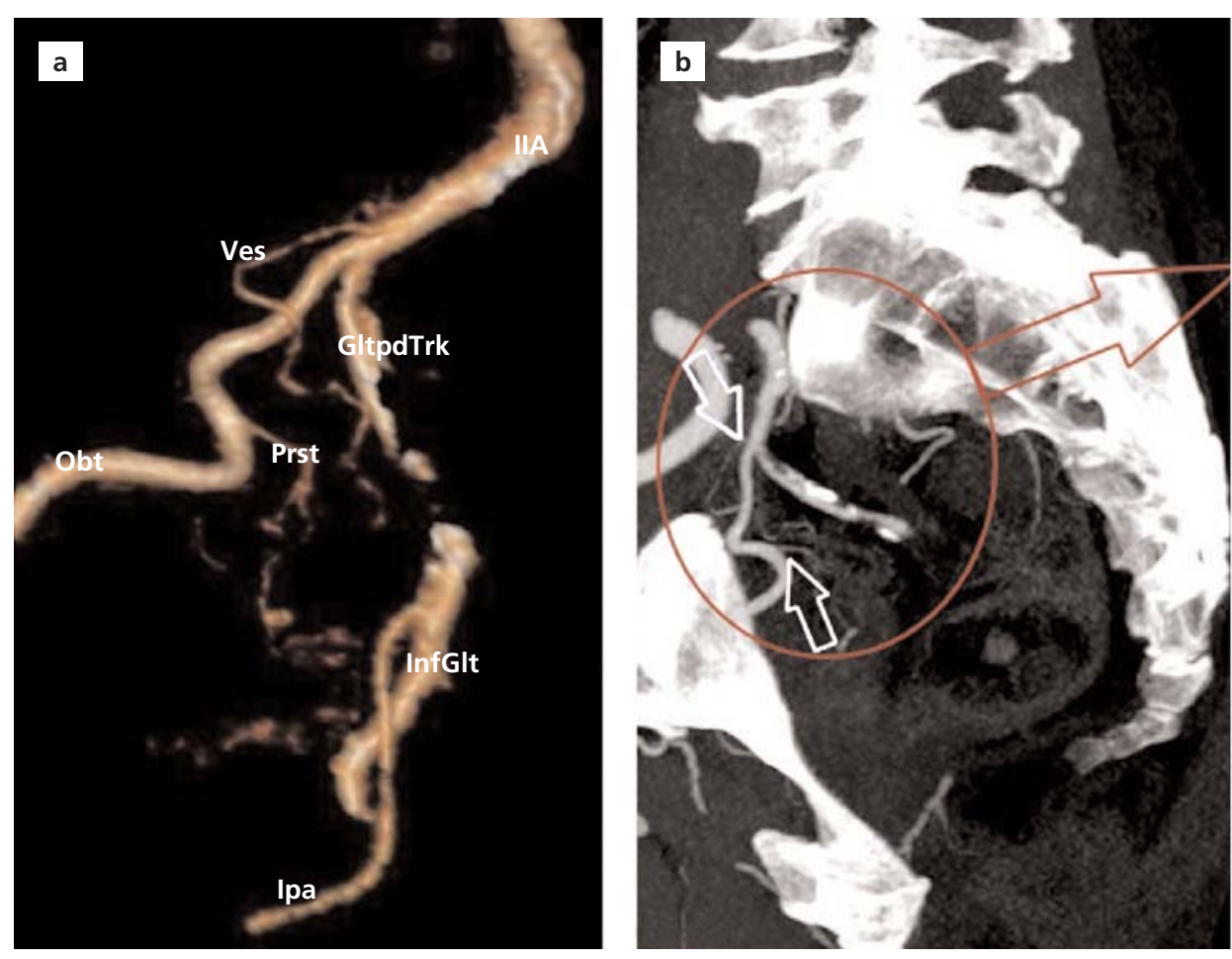

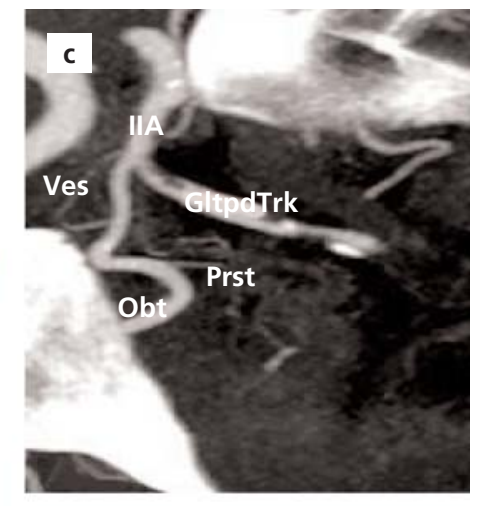

Figure 3. Imaging the internal iliac artery. (a) 3D rendered; (b) Curved MPR; (c) Spot image area curved MPR. GltpdTrk: gluteopudendal trunk; IIA: internal iliac artery; InfGlt: inferior gluteal artery; Ipa: internal pudendal artery; Obt: obturator artery; Prst: prostatic artery; Ves: vesical artery. [Color figure can be viewed in the online issue, which is available at www.anatomy.org.tr] the gold standard method for depicting the pelvic vascular anatomy while planning the PAE. ${ }^{[5,13]}$

Bouissou and Talazac ${ }^{[14]}$ were the first to describe two cadaveric independent PAs on each pelvic side. They dissected 100 pelvic halves and defined a cranial PA supplying inner and cranial prostate gland and a caudal PA supplying the peripheral and caudal gland. Zhang et al. ${ }^{[10]}$ reported a $3.6 \%$ rate of two independent PAs of the pelvic side in their study. Two independent PAs may cause insufficient clinical outcomes after PAE and must be kept in mind. In our study, we found accessory prostatic arteries in 10 cases. This finding is coherent with the literature.

Clegg et al ${ }^{[15]}$ studied PA in 21 postmortem materials in 1955. They used radio-opaque medium to assist the arterial supply of the prostate. The radiographs of the dissected specimens were obtained. They found that the prostatic gland was supplied by prostatic branch of an artery that was named as prostato-vesical artery. They defined prostato-vesical artery as a well-defined trunk with a variable origin but this is not a current nomenclature. They also reported superior rectal artery giving rise to PA in $32.1 \%$ of the cases. In this study, we did not find any PA arising from the superior rectal artery.

Ambrosio et al ${ }^{[16]}$ studied with 40 pelvic halves in 1980 and found that PA origin was variable (arising from the inferior vesical, internal pudendal, umbilical, obturator, inferior gluteal or internal iliac artery). Similarly, in this study, we did not find any internal iliac artery giving rise to PA.

Moya et al ${ }^{[8]}$ studied on 10 adult pelvic sides from embalmed cadavers and 34 DSA pelvic angiographies. Fifty-eight PAs were evaluated in their study. They generated six types of PA origins using a total of 735 PAs including their data and previously published data. This study included different patients from different countries and did not present the variations of PA in a certain population. These data were obtained from cadaver dissections, DSA and cone beam CT. ${ }^{[8]}$

In this study, we evaluated 242 PAs of a single population by the same radiologist using CTA as a single procedure. According to the classification of PAs in the work of

Table 2

Origin of the left prostatic artery.

\begin{tabular}{lcc}
\hline Origin & $\mathbf{n}$ & Ratio (\%) \\
\hline Inferior vesical artery & 43 & 36 \\
Vesical trunk & 3 & 2.5 \\
Gluteo-pudendal trunk & 11 & 9.1 \\
Obturator artery & 16 & 13 \\
Internal pudendal artery & 38 & 31 \\
Inferior gluteal artery & 3 & 2.5 \\
\hline
\end{tabular}


Moya et al. ${ }^{[8]}$ Type I, indicated anterior division of internal pudendal artery to give rise to $\mathrm{PA}$, and Type IV, which presents internal pudendal artery as the origin site of PA, were the most common types.

In the present study, the most common origin of the PA was the inferior vesical artery arising from the anterior division of the internal iliac arteries (Type I in Moya classification). The internal pudendal artery was the second most common site of origin for the PA in our population (Type IV in Moya classification).

In this study, there are differences from the previously published studies in the literature. We suggest that this discrepancy may depend on different patient populations from different countries. However, we need larger patient series to claim this hypothesis in terms of statistics, and this is one of limitations of our study.

\section{Conclusion}

It is very important to understand the PA anatomy prior to any surgical or interventional procedure related to the prostate gland. Conventional CTA is the gold standard for depicting PA anatomy, and should be considered as a first line option before surgical or interventional procedures.

\section{References}

1. Carnevale FC, Antunes AA, da Motta Leal Filho JM, de Oliveira Cerri LM, Baroni RH, Marcelino AS, Freire GC, Moreira AM, Srougi M, Cerri GG. Prostatic artery embolization as a primary treatment for benign prostatic hyperplasia: preliminary results in two patients. Cardiovasc Intervent Radiol 2010;33:355-61.

2. Garvey B, Türkbey B, Truong H, Bernardo M, Periaswamy S, Choyke PL. Clinical value of prostate segmentation and volume determination on MRI in benign prostatic hyperplasia. Diagn Interv Radiol 2014;20:229-33.

3. Mirakhur A, McWilliams JP. Prostate artery embolization for benign prostatic hyperplasia: current status. Can Assoc Radiol J 2017;68:84-9.

4. Pisco J, Bilhim T, Pinheiro LC, Fernandes L, Pereira J, Costa NV, Duarte M, Oliveira AGJ. Prostate embolization as an alternative to open surgery in patients with large prostate and moderate to severe lower urinary tract symptoms. Vasc Interv Radiol 2016;27:700-8.

5. Bilhim T, Casal D, Furtado A, Pais D, O'Neill JE, Pisco JM. Branching patterns of the male internal iliac artery: imaging findings. Surg Radiol Anat 2011;33:151-9.

6. Park BJ, Sung DJ, Kim MJ, Cho SB, Kim YH, Chung KB, Kang $\mathrm{SH}$, Cheon J. The incidence and anatomy of accessory pudendal arteries as depicted on multidetector-row CT angiography: clinical implications of preoperative evaluation for laparoscopic and robot-assisted radical prostatectomy. Korean J Radiol 2009;10:587-95.

7. Bilhim T, Tinto HR, Fernandes L, Martins Pisco J. Radiological anatomy of prostatic arteries. Tech Vasc Interv Radiol 2012;15:27685 .

8. Moya C, Cuesta J, Friera A, Gil-Vernet Sedo JM, ValderramaCanales FJ. Cadaveric and radiologic study of the anatomical variations of the prostatic arteries: a review of the literature and a new classification proposal with application to prostatectomy. Clin Anat 2017; 30:71-80.

9. Yamaki K, Saga T, Doi Y, Aida K, Yoshizuka M. A statistical study of the branching of the human internal iliac artery. Kurume Med J 1998;45:333-40.

10. Zhang G, Wang M, Duan F, Yuan K, Li K, Yan J, Chang Z, Wang Y. Radiological findings of prostatic arterial anatomy for prostatic arterial embolization: preliminary study in 55 Chinese patients with benign prostatic hyperplasia. PLoS One 2015;10:e0132678.

11. Venuti JM, Imielinska C, Molholt P. New views of male pelvic anatomy: role of computer-generated 3D images. Clin Anat 2004;17: 261-71.

12. Ding HM1, Yin ZX, Zhou XB, Li YB, Tang ML, Chen SH, Xu DC, Zhong SZ. Three-dimensional visualization of pelvic vascularity. Surg Radiol Anat 2008;30:437-42.

13. Desai H, Yu H, Ohana E, Gunnell ET, Kim J, Isaacson A. Comparative analysis of cone-beam CT angiogram and conventional CT angiogram for prostatic artery identification prior to embolization. J Vasc Interv Radiol 2018;29:229-32.

14. Boussou H, Talazac A. Arterial vascularization of the normal and the pathological prostate. Ann Anat Pathol (Paris). 1959;4:63-79.

15. Clegg EJ. The arterial supply of the human prostate and seminal vesicles. J Anat 1955;89:209-16.

16. Ambrósio JD, De Almeida JS, De Souza A. Origin of prostatic arteries in man. Rev Paul Med 1980;96:52-5.
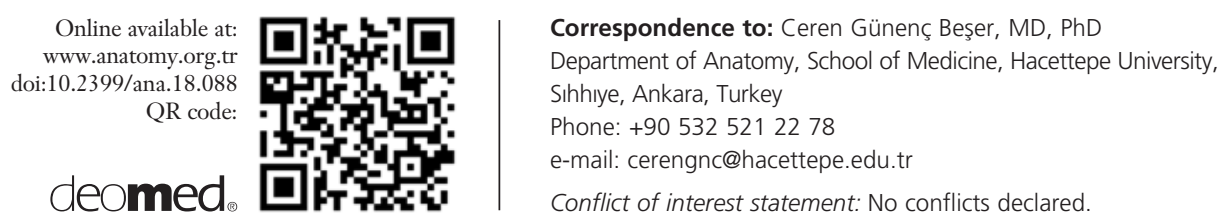

This is an open access article distributed under the terms of the Creative Commons Attribution-NonCommercial-NoDerivs 3.0 Unported (CC BY-NCND3.0) Licence (http://creativecommons.org/licenses/by-nc-nd/3.0/) which permits unrestricted noncommercial use, distribution, and reproduction in any medium, provided the original work is properly cited. Please cite this article as: Çelebioğlu EC, Akkaşoğlu S, Çalışkan S, Günenç Beşer C, Sancak T. Evaluation of the prostatic artery origin using computed tomography angiography. Anatomy 2018;12(3):140-144. 